\title{
Alfabetización digital universitaria en estudiantes de ingeniería en tiempos de pandemia
}

\section{University digital literacy in engineering students times of pandemic}

\author{
Jessica Paola Palacios Garay* (D) Doris Elida Fuster Guillen (D), Jhonny Richard Rodríguez

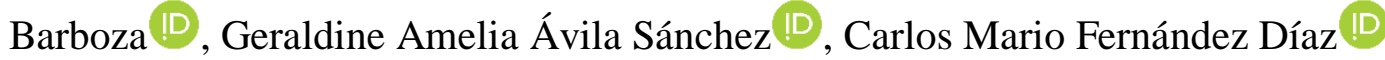 \\ Universidad Nacional Mayor de San Marcos, Perú \\ *Corresponding author (e-mail): jpalaciosg@ unmsm.edu.pe
}

(recibido/received: 25-julio-2021; aceptado/accepted: 15-septiembre-2021)

\begin{abstract}
RESUMEN
El propósito de este estudio radica en contextualizar la alfabetización digital de los estudiantes universitarios dentro de la actual pandemia, tomando en cuenta el desarrollo de habilidades y retos de esta nueva era digital. Analizar, además, sus alcances y límites para el desarrollo de los estudiantes, quienes siguen con sus estudios profesionales a pesar del confinamiento. Fue un estudio cuantitativodescriptivo, con variables sociodemográficas (edad, sexo, tiempo de conectividad); la muestra constó de estudiantes de ingeniería de una universidad pública: 213 hombres $(64,5 \%)$ y 117 mujeres $(35,5 \%)$ del quinto ciclo de carrera. Se utilizó el instrumento de Area (2014). La alfabetización digital y la formación de la ciudadanía del siglo XXI. Concluimos que la alfabetización digital seguirá ampliándose cada vez más, ya que la educación se basa en la virtualidad a causa de la pandemia de covid-19, de modo, que esta modalidad seguirá ampliándose para que los estudiantes continúen con sus estudios superiores con normalidad, desarrollando conocimientos tecnológicos.
\end{abstract}

Palabras claves: alfabetización digital, tecnología, internet, virtualidad, covid-19

\begin{abstract}
The purpose of this study is to contextualize the digital literacy of university students within the current pandemic, taking into account the development of skills and challenges of this new digital era. Analyze, in addition, its scope and limits for the development of students, who continue with their professional studies despite confinement. It was a quantitative-descriptive study, with sociodemographic variables (age, sex, time of connectivity); The sample consisted of engineering students from a public university: 213 men $(64.5 \%)$ and 117 women $(35.5 \%)$ from the fifth cycle of the degree. The Area instrument (2014) was used. Digital literacy and the formation of citizenship of the XXI century. We conclude that digital literacy will continue to expand more and more, since education is based on virtuality due to the covid-19 pandemic, so that this modality will continue to expand so that students continue with their higher studies normally, developing technological knowledge.
\end{abstract}

Keywords: digital literacy, technology, internet, virtuality, covid-19

\section{INTRODUCCIÓN}

La pandemia a raíz de la covid-19 ha traspasado los ámbitos relativos a la sociedad, técnicos, educativos y poblacionales, y dejó demostrado que la alfabetización digital es un valor impulsor del 
progreso sostenible, que confiere al mismo tiempo una superior intervención de los individuos en el sitio laboral, refuerza la salud, disminuye la pobreza y aumenta las posibilidades de progreso a lo largo del vivir. Además, la tecnología mejorada y el acceso total a internet, acentuado en una era de aislamiento, ofrecen ventajas. Entretanto, el aumento de su utilidad y empleo de parte de los individuos refiere, además, un aumento de riesgo a las advertencias del medio ambiente, a lo que es determinante estar vigilante e informado (Organización de las Naciones Unidas para la Educación, la Ciencia y la Cultura (Unesco, 2020).

La alfabetización digital, mencionada por la Unesco (2020), recorre un proceso que tiene como objetivo un conjunto de habilidades fundamentales para gestionar nuevas herramientas, programas, dialectos y redes. Si bien es cierto es un área importante, no es lo mismo que el tema de ciudadanía digital, es decir, observar, desde una perspectiva macro, que esta forma de enseñanza es un pilar permanente que se encuentra en constante cambio y que integra nuevas acciones y actitudes hacia las nuevas tecnologías, así como nuestros deberes y derechos.

Para Salado et al. (2020), la alfabetización digital es un recurso constante que no solo consiste en adquirir la destreza de escribir y leer, entender una noción o emplear una herramienta. Es por eso que se debe considerar la inclusión de la alfabetización digital en el plan de estudios en todo nivel educativo, y en la formación universitaria, es primordial debido a que, según Salado et al. (2020), es un medio para adquirir enseñanzas fundamentales para saber y emplear apropiadamente las tecnologías de información y contestar analíticamente a los incentivos y requerimientos de un contexto informacional cada vez más difícil.

Así mismo, la alfabetización digital conlleva tomar acuerdos tecnológicos con la finalidad de discernir el campo de estudio y desde qué óptica se abordará este (Salado et al., 2020). En la universidad, donde el discernimiento se comienza a profundizar, es primordial que los alumnos presenten esta destreza. Esto debido a que, al poseer esta habilidad podemos manejar un pensamiento crítico y analítico, pues al acceder y analizar distintas fuentes de información nos convertimos en personas más reflexivas y analíticas. Asimismo, esta herramienta no solo sirve en el ámbito educativo, sino también a nivel personal, pues ayuda a ocupar nuestro tiempo en cosas que realmente nos benefician en diferentes aspectos de la vida.

El objetivo de la investigación es contextualizar la alfabetización digital en estudiantes universitarios durante la pandemia a causa de la covid-19. Asimismo, busca ensartar al lector en el ámbito de la alfabetización digital de una manera simple, pero consolidada en diferentes fuentes bibliográficas reconocidas. Para ello emplearemos una metodología de investigación cualitativa, es decir, se situará la alfabetización digital en estudiantes universitarios en el marco de la pandemia. Consideramos que esta investigación en la actualidad es un aporte importante al conocimiento del fenómeno, pues debido a esta pandemia debemos estar informados respecto al tema. Con respecto a la teoría de la conectividad, Siemens (2004) mencionó que la tecnología impactará en las teorías de aprendizaje como el conductismo, el cognitivismo y el constructivismo, con el objetivo de mejorar su experiencia y aprendizaje en el mundo del conocimiento digital, que del mismo modo comparte la ideología con López y Escobedo (2021).

\section{REVISIÓN DE LITERATURA}

\section{La educación superior en el contexto de la pandemia}

Los representantes sociales que forman parte del sistema educativo - alumnos, docentes, trabajadores administrativos y dirigentes-, al igual que en otros ámbitos sociales que se relacionan con instituciones universitarias y superiores, escuelas y centros investigativos, se han visto en la obligación de enfrentar con prisa e ingenio el desafío de reajustar sus actividades sustanciales a fin de continuar asumiendo los desafíos y dificultades que enfrentan desde hace años (Ordorika, 2020). 
Según Ordorika (2020), el impacto de la pandemia en la educación superiora a nivel mundial fue mayúsculo porque, conforme a una encuesta internacional realizada por International Association of Universities (2020), para el $80 \%$ de instituciones de educación superior el impacto radicó en el aumento de inscripciones de estudiantes nacionales e internacionales; asimismo, el $48 \%$ de los gobiernos de los respectivos países tomaron medidas para que los estudiantes puedan completar sus estudios superiores. Por otro lado, las instituciones expresan que la educación virtual ha sustituido a la educación presencial, de modo que este gran cambio ha puesto muchos retos tecnológicos, pedagógicos y de competencias. La encuesta también muestra que el $80 \%$ de las actividades de investigación fueron afectadas, ya que se cancelaron vuelos internacionales, conferencias científicas y la cancelación de diversos proyectos.

Ordorika (2020), además, hace referencia a que la abolición de clases presenciales en todo el mundo tuvo que ser reemplazada por la educación virtual, lo que generó muchas dificultades; muy aparte de las tecnológicas, también incrementó la exclusión y marginación. Es por ello por lo que la Unesco (citado en Ordorika, 2020) menciona que el mundo no estaba preparado para todo eso: de la noche a la mañana las escuelas y universidades cerraron y de forma apresurada tuvieron que implementar cambios. De la misma manera, hace referencia a que mínimamente uno de cada dos hogares cuenta con banda ancha de internet, lo que afecta a la accesibilidad para seguir con los estudios. Todo esto resume en que el nuevo modelo educativo virtual ha causado muchos cambios en la educación superior.

\section{La educación superior en diferentes países en tiempos de pandemia}

Rodicio-García et al. (2020), en su investigación «Brecha digital en estudiantes españoles ante la crisis de la covid-19», tratan de conocer cómo los estudiantes de diferentes niveles educativos están viviendo la educación no presencial en tiempos de pandemia. La muestra estuvo conformada por 593 estudiantes (76,4\% mujeres, $22,6 \%$ hombres y el $1 \%$ que no especificó género); las edades oscilan entre los 18 y los 79 años. De la misma manera, cursan enseñanza superior (61,7\%), formación profesional $(17,9 \%)$, bachillerato $(15,9 \%)$ y enseñanza secundaria obligatoria $(4,6 \%)$. Se les aplicó el cuestionario de tecnoestrés elaborado por Wang \& Li (2019). Mediante esta investigación, se llegó a conocer que la mayoría de los estudiantes españoles cuentan con dispositivos tecnológicos como celulares, laptops o tabletas junto con la conexión a internet para recibir clases virtuales. Sin embargo, el Instituto Nacional de Estadística (INE, 2019, citado en Rodicio-García, 2020) menciona que el acceso a los recursos digitales no es el mismo en todos los hogares, ya que algunas localidades de 10000 habitantes en zonas rurales no cuentan con bandas anchas de internet.

Asimismo, en un estudio de revisión, Rogowska et al. (2020) mostraron que los efectos psicológicos adversos del aislamiento a menudo incluyen síntomas de confusión, ira y trastorno de estrés postraumático en la vida universitaria, así como una mayor prevalencia de síntomas de ansiedad y depresión en la población general. Las reacciones psicológicas y conductuales más comunes en aislamiento son irritabilidad, nerviosismo, depresión, trastornos emocionales, tristeza, culpa, agotamiento, aburrimiento, insomnio, falta de información, desesperación e indecisión, alienación de los demás, deterioro del rendimiento laboral y problemas económicos. Al comparar la actividad de los usuarios en Weibo (una red social popular en China) antes y después del brote de covid-19 en China se encuentra que las emociones negativas como la ansiedad, la depresión y la ira han aumentado, y también son más sensibles a los riesgos sociales. También se observó una disminución de las emociones positivas y de la satisfacción con la vida. Todo eso gracias a una investigación realizada en China durante la pandemia de covid-19 en la cual se encontró que el nivel de salud mental era bajo y la proporción de ansiedad, depresión y consumo de alcohol era más alta de lo habitual.

\section{La alfabetización digital}

La expresión alfabetización digital se utilizó a fines de la década de 1990, y era definida como la capacidad de comprender y usar información en múltiples formatos de múltiples fuentes cuando se 
presenta en una computadora. Asimismo, Díaz y Loyola (2021) hacen énfasis en captar la información en diversos formatos haciendo más uso del pensamiento crítico que de las habilidades de comunicación e informática, de modo que las personas puedan desarrollar ingenio en la tecnología, ya que en pleno siglo XXI es muy utilizada por todo el mundo (Díaz y Loyola, 2021).

Según la Unesco (2020), la alfabetización digital implica adquirir habilidades básicas en la gestión de tecnología, uso de equipos y nuevas formas de comunicación; de modo que se van incorporando al comportamiento y a las actitudes de cada persona entorno a una vida digitalizada. Los elementos digitales en estos tiempos de pandemia son muy importantes, pues rigen en toda la sociedad porque deben de estar en continuas actualizaciones digitales para establecer la interconectividad y virtualidad. De la misma manera, Tang y Chaw (2016, como se citó en Díaz y Loyola, 2021) presentan una amplia definición para ello, refiriéndose como el conocimiento y la capacidad que las personas poseen para utilizar las herramientas digitales; así como es necesario que sepan identificarlas, acceder a ellas, evaluar y sintetizar los recursos digitales para buscar la interconexión entre todo el mundo.

Como se sabe, la alfabetización digital incluye el conocimiento de varias fuentes de información digital para su uso. Por ello, García (2017) hace referencia a cinco dimensiones. En primer lugar, tenemos a la dimensión instrumental: el conocimiento y habilidades prácticas para el uso del hardware y software; la dimensión de inteligencia cognitiva concierne a los conocimientos y habilidades cognitivas que permiten la búsqueda, selección, análisis, interpretación y recreación; la dimensión de comunicación social está relacionada con las habilidades comunicativas a través de las TIC, también incluye comportamientos y actitudes sociales positivas como el respeto, trabajo cooperativo con los demás; dimensión de axiología trata de sensibilizar sobre el impacto de las TIC es de gran importancia en el entorno cultural y político de la sociedad junto con la adquisición de valores morales y democráticos relacionados con el uso información. Y por último, la dimensión emocional, en donde intervienen los sentimientos y emociones en la experiencia digital, de modo que surja un equilibrio afectivo en el uso de las TIC.

García (2017) hace referencia a ciertas habilidades de desarrollo muy necesarias para el buen manejo de la información digital, en donde los individuos deben adquirir y desarrollar capacidades de uso necesario durante toda la vida para la resolución de inconvenientes. Así como en el entorno educativo se necesita una pedagogía activa centrada en el alumno, basada en los recursos y en la solución de inconvenientes en el entorno digital, de igual manera en el entorno sociolaboral se debe tener dominio del estudio, administración, recuperación y evaluación de la información electrónica. En el entorno económico, ocupaciones de servicio, apoyadas en las tecnologías para un veloz desarrollo como mejorar la infraestructura de ingreso a las multiplataforma para asegurar la ciberseguridad de la información. De modo que, si los individuos están preparados tecnológicamente, no tendrán ningún problema durante los próximos años, ya que nos encontramos en una era digitalizada.

La utilidad de la alfabetización digital en los estudiantes, según Tistar (2006, como se citó en García, 2017), promueve que tengan un mejor manejo del software y del hardware, sean flexibles durante jornadas académicas, tengan mayor acceso a fuentes científicas y académicas, desarrollen la creatividad, sean capaces de contextualizar la información de la red, estén actualizados con la nueva información y estén interconectados con todo el mundo y de esa forma puedan tener más contacto de personas de todo el mundo.

Dado que la tecnología está en constante evolución a medida que pasan los años, se considera sumamente importante el desarrollo de ciertas habilidades que, según Carretero et al. (2017), en el Marco de Competencias Digitales para los Ciudadanos (The Digital Competence Framework for Citizens-DigComp), destacan cinco competencias y 21 componentes que la conforman.

La primera competencia es la alfabetización en información y datos en donde se desarrolla la navegación, búsqueda, filtrado de datos, información y contenido digital; evaluación de datos e información digital y gestión de datos y contenido digital. La segunda competencia es comunicación y elaboración, tomando en cuenta la interacción a través de tecnologías digitales, compartir a través de 
tecnologías digitales, el compromiso con la ciudadanía a través de tecnologías digitales, colaboración a través de tecnologías digitales, netiquette ('etiqueta digital') y gestión de la identidad digital. La tercera competencia corresponde a la creación de contenido digital en donde intervendría el desarrollo de esta, así como la elaboración, el derecho de autor y licencia, y la programación del lanzamiento del contenido. En cuanto al cuarto componente, pertenece a la seguridad por medio de la protección de los dispositivos, privacidad, bienestar del personal y del medio ambiente. Por último, el quinto componente es la solución de problemas tomando en cuenta la solución de los problemas técnicas, identificación de la tecnología, uso creativo y la identificación de las brechas digitales en todo el mundo.

\section{Los retos de la alfabetización digital}

El 8 de septiembre de 1967, la Organización de las Naciones Unidas para la Educación, Ciencia y Cultura (Unesco) (s. f.) estableció el Día Internacional de la Alfabetización, que tuvo como finalidad despertar el interés sobre la lectura y la escritura en las comunidades internacionales para que esta sea tomada como indispensable y se considere un derecho humano. Para Del Moral (2015, como se citó en Rojas et al., 2018), los profesores tienen la necesidad de adoptar las nuevas herramientas tecnológicas, ya que las prácticas educativas se encuentran abiertas a diversas posibilidades para así instruir y aprender desde el marco digital, todo ello han puesto nuevos retos y formas de aceptar el proceso educativo de manera virtual, ya que ahora los estudiantes adquieren desde temprana edad conocimientos a través de nuevas tecnologías de información y comunicación (TIC).

El surgimiento de la tecnología de información y comunicación modificó la manera de hacer las cosas y de ver el mundo. Estos cambios hoy se ven en el campo económico, político o social, y hasta cierto punto han determinado un crecimiento en el campo de la educación, especialmente en instituciones de educación superior (IES), porque generan mucho conocimiento, y luego se encargarán de difundir en el aspecto social de distintas formas. Aunque no son solo estas instituciones las que tienen la capacidad de generar conocimiento, su desempeño en un nuevo entorno de la sociedad es fundamental porque en ellas está el cumplir con sus responsabilidades de satisfacer las necesidades de su entorno (Aguilar et al., 2020).

Esta necesidad de alfabetización digital va a generar un liderazgo personal u otros aspectos como el revisar y enviar nuevos datos personales a los docentes universitarios, porque son los responsables de proporcionar el conocimiento y desarrollar habilidades y actitudes requeridas. Pero para que el maestro pueda proporcionar este tipo de resultados, es necesario asegurar un cierto puntaje alto que les permita un uso correcto y eficaz de las TIC, hasta llegar a cierto punto de dominar, comprender, evaluar y utilizar las TIC, de tal manera que se dé una capacidad de aprender en los alumnos, los cuales serán modelados en el conocimiento de que estas herramientas generan una amplia gama de alternativas tecnológicas (Carneiro et al., 2019).

Según García et al. (2020), la crisis sanitaria causada por la covid-19 ha obligado al sistema educativo a buscar reformas cambiando las aulas y clases de manera presencial por habitaciones en casa, y a mantener la educación gracias a la tecnología que nos permite seguir conectados gracias a la variedad de presentaciones como las computadoras, tabletas, televisión y radio. Es por esto que se ha tenido que poner a disposición de docentes variedad de recursos para agilizar la enseñanza a través de estos medios. Pero a pesar de lo elaborado de este sistema, también se puede observar dificultades para el alumno, ya que algunas familias no cuentan con los dispositivos necesarios para cubrir la educación virtual.

Para Shopova (2014), la competencia digital está determinada por la Unión Europea como una de las ocho competencias claves para el aprendizaje permanente, que debido a su versatilidad le permite adquirir otras habilidades claves; por ejemplo, matemáticas, aprender a aprender, creatividad, y asegura la participación activa en la sociedad y economía, según el Parlamento Europeo y Consejo (2006, como se citó en Shopova, 2014). Esta competencia implica «el uso seguro y crítico de la Information Society Technology (IST) para el trabajo, el ocio y la comunicación». Esto significa un 
gran incremento de posibilidades para una alfabetización digital con mejores resultados. Debido a la pandemia por covid-19, hemos tenido que mejorar nuestros medios de comunicación, teniendo como reforma una educación totalmente digitalizada, en la cual se han presentado grandes retos. Por otro lado, Novomisky et al. (2020) alega que ahora que los estudiantes usan las redes sociales con mayor regularidad, los docentes tendrán la oportunidad de promover su uso como TAC (tecnologías para el aprendizaje y el conocimiento) y como TEPP (tecnologías para el empoderamiento y la participación). Díaz y Loyola (2021) nos dicen que los objetivos para una educación virtual adecuada sería que se logre diferenciar las ideas que tienen las competencias digitales tomando en cuenta las fortalezas y debilidades de los maestros y alumnos, para así relacionarlas con la alfabetización y mejorarlas, así mismo reflexionar sobre el efecto educativo que ha causado las clases virtuales para que se pueda brindar diferentes estrategias y lograr el crecimiento y la elaboración de un plan adecuado.

Para Valdivia (2020), el problema de la alfabetización digital es que los docentes no tienen una formación adecuada ni específica para trabajar con jóvenes y adultos, por ellos vemos los problemas al momento del desarrollo de las clases, ya que en general su formación para ser docentes no incluye el uso de dispositivos, herramientas digitales ni se plantean propuestas que impliquen aprender utilizando la tecnología, por ello las limitaciones de sus conocimientos causan dificultad y más aún ahora que la educación es $100 \%$ virtual.

Asimismo, para Novomisky et al. (2020), el vínculo del docente con el estudiante va más allá de brindar contenidos, tareas y correcciones, sino este debería generar espacios en aulas virtuales, redes sociales o cualquier otro canal de comunicación a distancia que se adapte para compartir conversaciones que permitan fortalecer los lazos, como pueden ser los foros $\mathrm{u}$ otros espacios, con el fin de acompañar y sostener a los estudiantes.

Por otro lado, de acuerdo con Delgado (2020), se han improvisado medidas para el aprendizaje y alfabetización en tiempo de covid-19, pues se tuvieron que idear implementos, entre ellos algunos equipos directivos los cuales fortalezcan y afiancen la educación, como la creación del programa «Aprender en casa», desarrollado en el confinamiento del 2020 a raíz de la pandemia de covid-19. Esto puso en evidencia la desventaja ante los desafíos en todos ámbitos de México, donde se optó por un replanteamiento de la enseñanza, teniendo como objetivo el avance en la alfabetización centrado en el adecuado uso de las herramientas digitales implicando la integración de estas en el proceso educativo, poniendo énfasis en las necesidades y particulares de las diversos contextos del país, para lograr este objetivo se tuvo que trabajar con maestros ya que estos eran los que tuvieron un exceso de carga laboral debido a las nuevas implementaciones por la pandemia, teniendo a su cargo grupos números de entre 25 a 45 estudiantes, por esto se les proporcionó un acompañamiento y apoyo ante la posibilidad del estrés, por otro lado, fortaleciendo su comunicación con el alumnado vía WhatsApp, a través de materiales en YouTube y textos interactivos en Word y PDF.

Para Llorens et al. (2021), Chile, en el ámbito de la educación, está sujeto a diferentes cambios en los cuales se realizan estrategias e innovaciones con las cuales se busca adecuarse a los cambios que acontecen la globalización. Al igual en otros ámbitos, la sociedad chilena se ha visto en la necesidad de mejorar e implementar educativas, afianzando la comunicación a distancia mediante tecnológicas digitales tratando de crear una conectividad igualitaria y efectiva, poniéndolo al alcance de los estudiantes, los cuales debieron pasar por un proceso de adaptación a las nuevas condiciones como son las aulas virtuales. Es por esto por lo que se realiza una investigación con la población estudiantil, para medir su desempeño ante esta coyuntura, en los cuales demuestran que el 50\% de los estudiantes pueden manejar el ámbito digital a partir de funciones como navegar por internet, la realización y entrega de trabajos escolares, por otro lado existe un $47 \%$ posee complicaciones para la conectividad, que van desde falta de implementos para conectarse ya sea cámara, laptop, entre otras hasta la falta de conectividad (internet). Por ello, la educación debe enfocarse en la igualdad de educación para todas las clases sociales, así se asegurará una alfabetización digital de calidad y con un acceso el cual cumpla con las necesidades de la educación. 
Según Mineo y Costa (2021), la alfabetización en la coyuntura actual presenta características como la inestabilidad e incertidumbre debido a la pandemia por covid-19, teniendo impacto en los ámbitos social, político y educativo, en los que el «medio de solución» es el uso intenso de las tecnologías digitales, poniendo a Brasil en una situación donde se trata de tener como objetivo la relación e integración entre sociedad, alfabetización, tecnología y educación, mostrando gran preocupación por parte de los profesores sobre la existencia de una variedad de nuevos conceptos, reglamentos educativos, los cuales se han tenido que someter a cambios, planteando una serie de desafíos los cuales aportarían a la culminación del año académico.

\section{Alfabetización digital y la vida universitaria en tiempos de pandemia}

Como bien resalta Ayala (2020), en el entorno educativo la pandemia ha traído una forzosa forma virtual que requiere una adaptación adecuada, ya sea por parte del docente como también por parte del alumno. Esto permite que permanezcan activos en el sistema educativo universitario. También abarca a las materias cuyo contenido se refiere a lo teórico y didáctico, en las cuales se han implementado instrumentos tecnológicos que permitieron acceder a un aprendizaje no presencial y adaptarse a los nuevos cambios que se han presentado. La concurrencia tecnológica permitió la comunicación a través de los chats, plataformas virtuales, correo electrónico o la plataforma Moodle. La escena más controversial es la que corresponde a las prácticas profesionales y los talleres, donde lo virtual y el uso tecnológico habilitado no reemplaza la imprescindible interacción profesional que se da al estar frente a un salón de clase.

Asimismo, Gil-Villa et al. (2020) mencionan que existe una abertura digital que deja fuera a demasiadas personas. Existen países donde el acceso a internet no llega a la mitad de población; por ende, no pueden conectarse y así no puede asistir a clases virtuales. El aprendizaje ha cambiado tanto en su contexto físico, pues ahora es el hogar de cada quien el espacio cultural y académico. Esto puede mejorar o empeorar el proceso de aprendizaje virtual, a comparación con el aprendizaje presencial, la cual se tenía antes de la pandemia, pues algunos padres no cuentan con la capacidad para ayudar o enseñar a sus hijos. Hay que tener en cuenta que los universitarios que tienen hermanos menores tienen que asimilar el rol de profesor y ayudarlos con sus deberes. A raíz de la exclusión social, se puede presentar desmoralización y decepción. Cabe resaltar que también hay cosas positivas a causa de la modificación del uso del papel, por plataformas de internet, como las redes de afinidad. Dicho encierro ha traído consigo una convivencia complicada o problemática, universitarios a raíz del confinamiento expresan inquietud, ansiedad, frustración, dificultad para concentrarse y por ello son propensos a pelear o mostrar mal humor.

\section{MÉTODOS}

\section{El estudio se desarrolló bajo el enfoque cuantitativo}

El tipo de la presente investigación es sustantiva. Sánchez et al. (2018) sostuvieron que la investigación sustantiva es aquella que describe y explica los hechos acontecidos; por ende, se considera a esta investigación que es de tipo sustantiva debido a que tiene por finalidad explicar los sucesos.

El nivel de la presente investigación es descriptivo debido a que tiene por característica describir, detallar y explicar los hechos más relevantes de un evento que se va a investigar (Sánchez et al., 2018). La técnica es la forma como se recopila la información y así de esa forma poder ser analizada (Hernández y Mendoza, 2018). Se utilizó la encuesta, cuyo instrumento fue el cuestionario de Area (2014). La alfabetización digital y la formación de la ciudadanía del siglo XXI.

Sánchez et al. (2018) sostuvieron que la unidad de análisis era un grupo que posee cualidades únicas y especiales que se tomará en cuenta en una evaluación y, en este caso, se considera como unidad de 
análisis a los estudiantes universitarios de la universidad pública, los cuales fueron 330 de ingeniería del quinto ciclo: 213 hombres $(64,5 \%)$ y 117 mujeres $(35,5 \%)$.

\section{RESULTADOS}

Tabla 1. Niveles de las dimensiones de la variable alfabetización digital

\begin{tabular}{lllllllll}
\hline \multirow{2}{*}{ Niveles } & \multicolumn{2}{c}{ Instrumental } & \multicolumn{2}{c}{ Cognitiva } & \multicolumn{2}{c}{ Comunicativa } & \multicolumn{2}{c}{ Axiológica } \\
\cline { 2 - 9 } & $\mathrm{f}$ & $\%$ & $\mathrm{f}$ & $\%$ & $\mathrm{f}$ & $\%$ & $\mathrm{f}$ & $\%$ \\
\hline Desfavorable & 172 & 52,1 & 156 & 47,3 & 154 & 46,7 & 177 & 53,6 \\
Regular & 72 & 21,8 & 94 & 28,5 & 101 & 30,6 & 75 & 22,7 \\
Favorable & 86 & 26,1 & 80 & 24,2 & 75 & 22,7 & 78 & 23,6 \\
Total & 330 & 100,0 & 330 & 100,0 & 330 & 100,0 & 330 & 100,0 \\
\hline
\end{tabular}

En la tabla 1 se encontró que el 52,1\% percibió un nivel desfavorable; el 21,8\% percibió nivel regular; el 26,1 \% percibió nivel favorable de la dimensión instrumental de la alfabetización digital. El $47,3 \%$ percibió un nivel desfavorable; el 28,5\% percibió nivel regular, el 24,2\% percibió nivel favorable de la dimensión cognitiva de la alfabetización digital. El 46,7\% percibió un nivel desfavorable; el 30,6\% percibió nivel regular; el 22,7\% percibió nivel favorable de la dimensión comunicativa de la alfabetización digital. Finalmente, el 53,6\% percibió un nivel desfavorable; el 22,7 \% percibió nivel regular; el 23,6 \% percibió nivel favorable de la dimensión axiológica.

Tabla 2. Niveles de la variable alfabetización digital según edad

\begin{tabular}{llllll}
\hline & & \multicolumn{3}{l}{ Alfabetización digital } & \\
\cline { 3 - 6 } & & Desfavorable & Regular & Favorable & Total \\
\hline Edad & Menor de 20 & 71 & 14 & 3 & 88 \\
& \multirow{2}{*}{$21-23$} & $21,5 \%$ & $4,2 \%$ & $0,9 \%$ & $26,7 \%$ \\
& \multirow{2}{*}{ De 24} & $23,6 \%$ & 36 & 21 & 135 \\
& \multirow{2}{*}{ Mayor de 25 } & 62 & $10,9 \%$ & $6,4 \%$ & $40,9 \%$ \\
& & $18,8 \%$ & 12 & 12 & 86 \\
\multirow{2}{*}{ Total } & 20 & $3,6 \%$ & $3,6 \%$ & $26,1 \%$ \\
& & $6,1 \%$ & 1 & 0 & 21 \\
& & 231 & $0,3 \%$ & $0,0 \%$ & $6,4 \%$ \\
\hline
\end{tabular}

En la tabla 2, se encontró que en los estudiantes menores 20 años el 21,5\% percibió un nivel desfavorable; el $4,2 \%$ percibió nivel regular; el $0,9 \%$ percibió nivel favorable de alfabetización digital. De 21 a 23 años, el 23,6 \% percibió un nivel desfavorable; el 10,9\% percibió nivel regular; el $6,4 \%$ percibió nivel favorable. Finalmente, los estudiantes mayores de 25 años el 6,1\% percibió un nivel desfavorable; el 0,3 \% percibió nivel regular de la variable alfabetización digital según edad.

Tabla 3. Niveles de la variable alfabetización digital según sexo

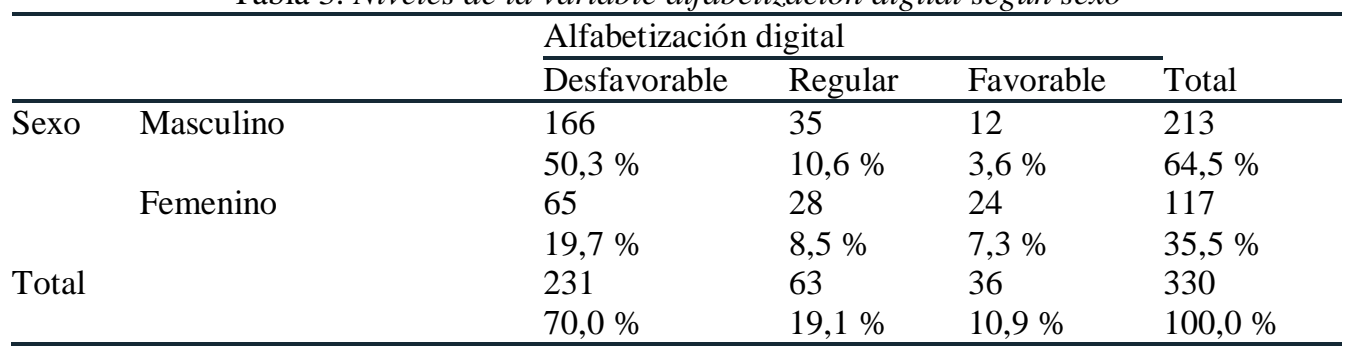

En la tabla 3, de los estudiantes de sexo masculino, el 50,3\% percibió un nivel desfavorable; el 10,6 \% percibió nivel regular; el 3,6 \% percibió nivel favorable de alfabetización digital. En cuanto a las de sexo femenino, el 19,7\% percibió un nivel desfavorable; el 8,5\% percibió nivel regular; el $7,3 \%$ percibió nivel favorable de la variable alfabetización digital según sexo. 
Tabla 4. Niveles de la variable alfabetización digital según tiempo de conectividad

\begin{tabular}{|c|c|c|c|c|c|}
\hline & & \multicolumn{3}{|c|}{ Alfabetización digital } & \multirow[b]{2}{*}{ Total } \\
\hline & & Desfavorable & Regular & Favorable & \\
\hline \multirow[t]{6}{*}{ Tiempo de conectividad } & Hasta 3 horas & 57 & 13 & 11 & 81 \\
\hline & & $17,3 \%$ & $3,9 \%$ & $3,3 \%$ & $24,5 \%$ \\
\hline & De 4 a 6 & 78 & 34 & 19 & 131 \\
\hline & & $23,6 \%$ & $10,3 \%$ & $5,8 \%$ & $39,7 \%$ \\
\hline & Más de 9 horas & 96 & 16 & 6 & 118 \\
\hline & & $29,1 \%$ & $4,8 \%$ & $1,8 \%$ & $35,8 \%$ \\
\hline \multirow{2}{*}{\multicolumn{2}{|c|}{ Total }} & 231 & 63 & 36 & 330 \\
\hline & & $70,0 \%$ & $19,1 \%$ & $10,9 \%$ & $100,0 \%$ \\
\hline
\end{tabular}

En la tabla 4, los estudiantes que manifiestan hasta tres horas de conectividad, el 17,3\% presentó un nivel desfavorable; el 3,9\% presentó nivel regular; el 3,3\% presentó nivel favorable de alfabetización digital y en cuanto a las estudiantes que manifiestan de 4 a 6 horas de conectividad; el 23,6 \% presentó un nivel desfavorable; el 10,3\% presentó nivel regular; el 5,8 \% percibió nivel favorable y en cuanto a los estudiantes que manifiestan más de 9 horas de conectividad; el 29,1\% presentó un nivel desfavorable; el $4,8 \%$ presentó nivel regular y el $1,8 \%$ percibió nivel favorable de la variable alfabetización digital según tiempo de conectividad.

\section{Alfabetización digital universitaria}

\section{DISCUSIÓN}

Los resultados demostraron que la alfabetización digital se encontró en nivel desfavorable en los estudiantes universitarios de ingeniería. Al respecto, George (2019) afirma que, debido a los nuevos desafíos que se presenta con respecto al uso de las tecnologías de la información y la comunicación (TIC), es necesario tener que adecuarnos y aprender sobre el uso e incluso el manejo de ellas; eso quiere decir que debemos de alfabetizarnos en lo relacionado con lo digital. Así también, podemos incluir a García (2017), quien mencionó que la alfabetización digital permite el avance y desarrollo de las habilidades con respecto al uso de los medios digitales para que de esa forma puedan emplear sus conocimientos en sus actividades y, ante ello, puedan solucionar las necesidades que se les pueda presentar en diferentes tipos de ámbitos.

Según Rangel y Peñalosa (2013), mencionaron que un individuo alfabetizado digitalmente estará capacitado en conocimiento y uso de ellas mediante las actitudes que presenten. Por otro lado (García et al., 2016), una persona se considera analfabeta digital cuando tiene un acceso limitado o un desarrollo bajo o nulo de las habilidades que le permitan interactuar en la red comunicativa que proporciona el uso de TIC.

En cuanto a las dimensiones de la alfabetización digital, la dimensión axiológica y la instrumental se encontraron en nivel desfavorables. Según Area (2014), la dimensión axiológica busca adquirir y desarrollar valores éticos, democráticos y críticos hacia la tecnología, así como la toma de conciencia de que las tecnologías de la información y comunicación no son asépticas ni neutrales desde un punto de vista social, sino que inciden significativamente en el entorno cultural y político de nuestra sociedad. En la dimensión instrumental está relacionada con el dominio técnico de cada tecnología (conocimiento práctico del hardware y del software que emplea cada medio), los resultados demuestran que los estudiantes desconocen de dicho domino técnico. Según Nawaz \& Kundi (2010), la alfabetización tecnológica, informática o computacional considera que los conocimientos y talentos son vitales para comprender y utilizar los sistemas informáticos y las aplicaciones informáticas de manera eficiente y eficaz en entornos no públicos.

\section{Alfabetización digital según edad}

De los estudiantes de 21 a 23 años, el 23,6 \% percibió un nivel desfavorable; el 10,9\% percibió nivel regular; el 6,4\% percibió nivel favorable; las personas con habilidades virtuales pueden desenvolverse 
de manera eficiente dentro del entorno multimedia, comunicarse satisfactoriamente en esas plataformas y trabajar de la mejor gracias al perfecto aprovechamiento de las oportunidades de la informática (Escandell, 2017). Por otra parte, la alfabetización digital es un conjunto de habilidades técnicas, procedimentales, cognitivas y socioemocionales, vital para permanecer, aprender y trabajar en una sociedad virtual más allá del uso correcto de dispositivos virtuales (Eshet-Alkalai, 2012; Fraser et al., 2013).

\section{Alfabetización digital según sexo}

Los estudiantes de sexo masculino, el 50,3\% percibió un nivel desfavorable, y en cuanto a las estudiantes de sexo femenino, el 19,7\% percibió un nivel desfavorable. Según Area (2014), la alfabetización es multimodal, es decir, el proceso alfabetizador debe desarrollar las competencias en múltiples lenguajes y medios, y debe partir de las experiencias culturales que el alumnado adquiere extraescolarmente. Vásquez-Cano et al. (2017) estudia las posibles diferencias de género respecto a las competencias digitales básicas para estudiantes de carreras de ciencias sociales de una universidad española. El estudio concluye que para el componente procesos de búsqueda de información en línea y presentación en línea los hombres muestran una mayor competencia; sin embargo, esto no ocurre para el resto de componentes, en los que se encuentra una equidad para ambos géneros. Asimismo, la alfabetización digital es todo el conjunto de habilidades que se utilizan para descubrir, manipular e interpretar adecuadamente las estadísticas a través del patrimonio neto; es decir, conocer el entorno de las TIC y reconocer suficientemente su funcionamiento para su correcto uso. Se piensa que los estudiantes con alfabetización digital pueden desenvolverse de manera eficiente dentro del entorno multimedia de internet, hablar satisfactoriamente sobre estos sistemas y pinturas con mayor éxito gracias al mejor aprovechamiento de las oportunidades de la informática (Escandell, 2017).

\section{Alfabetización digital según tiempo de conectividad}

En cuanto a los estudiantes que manifiestan más de nueve horas de conectividad, el 29,1\% presentó un nivel desfavorable; el 4,8\% presentó un nivel regular y el 1,8\% percibió nivel favorable de la variable alfabetización digital según tiempo de conectividad. El estudio de García (2019), en su estudio en estudiantes de ingeniería sobre el manejo de sus habilidades digitales y de las tecnologías de información y comunicación, concluyó que del total de estudiantes cuestionados, la mayoría tenía un nivel medio-bajo en cuanto a TIC y habilidades digitales se trataban, donde solo el $17,9 \%$ alcanzaba un nivel superior en el uso de estas a pesar de que todos los alumnos expresaron pasar al menos cinco horas semanales frente a su computadora para realizar tanto actividades académicas como actividades de exigencia social.

\section{CONCLUSIÓN}

La nueva modalidad en la que se desarrollaron las clases desde que la pandemia por covid-19 empezó tuvo varios retos, pues no estábamos preparados para asumir una educación virtual, aunque la tecnología es lo que nos mantiene informados y en comunicación constante, el poder adaptarnos a la nueva realidad fue muy difícil, así mismo uno de los problemas más notorios fue la falta de formación digital tanto de docentes como de alumnos lo que ha limitado su aprendizaje. Es por ello por lo que la alfabetización digital implica desarrollar habilidades para el consumo responsable de información para producir conocimiento; siempre y cuando se cumplan las normas específicas de la era digital, en cuanto a copyright, open data, hiperlenguajes.

La alfabetización digital es la nueva habilidad para la gestión de tecnología y forma de comunicación en este siglo XXI, ya que con las nuevas actualizaciones las personas pueden estar más conectadas a larga distancia. Asimismo, el desarrollo de competencias tecnológicas son un importante camino para la educación virtual, ya que los estudiantes deben estar cada vez más acostumbrados a esta era digitalizada para que no tengan ningún problema en el manejo de los dispositivos tecnológicos mediante el desarrollo de nuevas habilidades digitales. Una vez que los estudiantes universitarios se 
han alfabetizado digitalmente, el paso a seguir es fomentar el desarrollo de inteligencia digital, que promueve al cuidado de la persona para crear una identidad digital según los objetivos específicos de cada persona para privilegiar la protección y seguridad digital y el respeto de los derechos digitales de los demás, para fomentar la inteligencia emocional digital y asertiva en función de la construcción del grupo social.

La alfabetización digital ha sido un medio de enseñanza en los últimos años, el cual ha traído mejoras educativas al país, a partir del 2020 debido a la pandemia por covid-19; estas mejoras se han tenido que transformar e invertir pasando de ser un complemento de la educación a convertirse en la gran mayoría, ayudándose de dispositivos tecnológicos para cubrir con las necesidades de los estudiantes debido a esto; el estado ha recurrido medidas a través de páginas web, videos, aplicativos, textos en PDF (documentos de formato portátil), entre otros los cuales mejoren la calidad de enseñanza y sea posible una educación a distancia, de igual manera a nivel mundial, los diversos países han tenido que tomar diferentes medidas para afrontar esta situación.

\section{RECONOCIMIENTOS}

Esta investigación se realizó en el marco de la tarea universitaria que se viene realizando en tiempos de pandemia en una universidad pública de ingeniería, proyecto Alfabetización digital universitaria.

\section{REFERENCIAS}

Aguilar, F., Chamba, A. Hermann, A. Balladares, J., Henríquez, E., Oseda, D., Mendivel, R., Carhuachuco, E., Chávez, V., Jaramillo, L. y Simbaña, V. (2020). Filosofía de la innovación y de la tecnología educativa: Tomo III Innovación tecnológica en la educación. Editorial Abya-yala. https://dspace.ups.edu.ec/handle/123456789/19314.

Area, M. (2014). La alfabetización digital y la formación de la ciudadanía del siglo XXI Revista Integra Educativa, 7(3), 21-33. http://scielo.org.bo/pdf/rieiii/v7n3/v7n3_a02.pdf.

Ayala, A. (2020). Niveles de dificultad percibidos en el proceso de enseñanza-aprendizaje dentro del último año del profesorado universitario en biología (PUB) en el contexto de pandemia. Revista de $\begin{array}{llll}\text { Educación en } & \text { Biología, } & 2(2020), & \end{array}$ http://congresos.adbia.org.ar/index.php/congresos/article/view/203.

Carneiro, R., Toscano, J. y Díaz, T. (2019). Los desafíos de las TIC para el cambio educativo. Organización de Estados Iberoamericanos para la Educación, la Ciencia y la Cultura (OEI), 35-44. https://www.oei.es/uploads/files/microsites/28/140/lastic2.pdf.

Carretero, S., Vuorikari, R., y Punie, Y. (2017). DigComp 2.1: The Digital Competence Framework for Citizens: with eight proficiency levels and examples of use. Joint Research Centre. https://doi.org/10.2760/38842.

Delgado, M. (2020) Equipos directivos de educación primaria. Improvisar la alfabetización digital durante la cuarentena. En H. Casanova Cardiel (Coord.), Educación y pandemia: una visión académica (pp. 145-152). Universidad Nacional Autónoma de México, Instituto de Investigaciones sobre la Universidad y la Educación. https://www.iisue.unam.mx/nosotros/covid/educacion-ypandemia.

Diaz, D. y Loyola, E. (2021) Competencias digitales en el contexto covid-19: una mirada desde la educación. Revista Innova Educación, 3(1), 120-150. https://doi.org/10.35622/j.rie.2021.01.006.

Escandell, D. (2017). Alfabetismo digital en la enseñanza de segundas lenguas: espacios para una educación adaptada a las necesidades comunicativas de 38 nuestra época. Doblele revista de lengua y literatura. (3), 17-30. https://doi.org/10.5565/rev/doblele. 27. 
Eshet-Alkalai, Y. (2012). Thinking in the digital era: A revised model for digital literacy. Issues in Informing Science and Information Technology, 9, 257-276. http://iisit.org/Vol9/IISITv9p267276Eshet021.pdf.

Fraser, J., Atkins, L., \& Richard, H. (2013). DigiLit leicester. Supporting teachers, promoting digital literacy, transforming learning. Leicester City Council. http://lucyjca.co.uk/wpcontent/uploads/2016/10/digilit-leicester-report130625-final.pdf.

García , V. ; Aquino , S. P., y Ramírez , N. Á. (2016). Programa de alfabetización digital en México: 1:1. Análisis comparativo de las competencias digitales entre niños de primaria. CPU-e, Revista de Investigación Educativa, (23), 24-44. https://www.redalyc.org/articulo.oa?id=283146484003.

García, S. (2017). Alfabetización Digital. Razón y Palabra, 21(98), 66-81. https://www.redalyc.org/articulo.oa?id=199553113006.

García, S. (2019). Análisis de las competencias digitales de estudiantes de ingeniería de una universidad pública peruana. Hamutay, 6(3), 114-125. http://dx.doi.org/10.21503/hamu.v6i3.1852.

George, C. (2020), Alfabetización y alfabetización digital. Transdigital, 1(1). https://www.revistatransdigital.org/index.php/transdigital/article/view/15.

Gil-Villa, F., Urchaga Litago, J. y Sánchez Fernández, A. (2020). Percepciones y expectativas en el alumnado universitario a partir de la adaptación a la enseñanza no presencial motivada por la pandemia de covid-19: análisis de la percepción y repercusiones en la comunidad universitaria. Revista Latina de Comunicación Social, (78), 99-119. https://doi.org/10.4185/RLCS-2020-1470.

Hernández, R. y Mendoza, C. (2018). Metodología de la investigación Las rutas cuantitativa, cualitativa y mixta. Ciudad de México: McGraw-Hill Interamericana Editores, S. A. de C. V.

Llorens, A., Alarcon, J. y Brañes, J. (2021). Alfabetización digital y TIC en la educación secundaria en Chile: Diagnóstico en tiempo de pandemia. Interciencia: Revista de ciencia y tecnología de América, 46(4), 148-155. https://dialnet.unirioja.es/servlet/articulo?codigo=7933532.

López, E. y Escobedo, F. (2021). El conectivismo, el nuevo paradigma del aprendizaje. Revista Científica de Ciencias Sociales y Humanidades Desafíos, 12(1), 67-73. https://doi.org/10.37711/desafios.2021.12.1.259.

Mineo, W. y Costa, F. (2021). Rethinking Digital Literacy Practices and Educational Agendas in Times of Covid-19 Uncertainty. Brazilian Journal of Applied Linguistics, 21(2), 399-431. https://doi.org/10.1590/1984-6398202117285.

Nawaz, A., \& Kundi, G. M. (2010). Digital literacy: An Analysis of the Contemporary Paradigms. Journal of Science and Technology Education Research, 1(2), 19-29. https://academicjournals.org/journal/IJSTER/article-full-textpdf/68579321822.

Novomisky, S., Sujatovich, L. y Vestfrid, P. (2020). Alfabetización digital en tiempos de pandemia: conectividad, usos y representaciones de estudiantes de nivel inicial y primaria en institutos de formación docente. https://perio.unlp.edu.ar/wp-content/uploads/2020/05/Alfabetizaci\%C3\%B3ndigital-y-pandemia.pdf.

Ordorika, I. (2020). Pandemia y educación superior. Revista de la Educación Superior, 49(194), 1-8. http://resu.anuies.mx/ojs/index.php/resu/article/view/1120.

Organización de las Naciones Unidas para la Educación, Ciencia y Cultura. (s. f). Día Internacional de la Alfabetización. https://es.unesco.org/commemorations/literacyday . 
Organización de las Naciones Unidas para la Educación, la Ciencia y la Cultura. (17 de septiembre del 2020). Los nuevos desafios de la alfabetización. https://es.unesco.org/news/nuevos-desafiosalfabetizacion.

Rangel, A. \& Peñalosa, E. A. (2013). Alfabetización digital en docentes de educación superior: construcción y prueba empírica de un instrumento de evaluación.. Pixel-Bit. Revista de Medios y Educación, (43),9-23. https://www.redalyc.org/articulo.oa?id=36828074002.

Rodicio García, M. L., Ríos-de Deus, M. P., Mosquera-González, M. J., y Penado Abilleira, M. (2020). La brecha digital en estudiantes españoles ante la crisis de la covid-19. Revista Internacional de Educación para la Justicia Social, 9(3), 103-125. https://doi.org/10.15366/riejs2020.9.3.006.

Rogowska. A., Kuśnierz. C. y Bokszczanin. A. (2020). Examining anxiety, life satisfaction, overall health, stress and coping styles during the COVID-19 pandemic in a Polish sample of college students. Psychol Res Behav Manag, 2020(3), 797-811. https://doi.org/10.2147/PRBM.S266511.

Rojas, A., Salazar, A., Hilario, J., Mori, M. y Pasquel, A. (2018). Aplicación del módulo alfabetización digital y desarrollo de competencias digitales en docentes.Comuni@cción, 9(2), 101$110 . \quad$ http://www.scielo.org.pe/scielo.php?script=sci_arttext\&pid=S2219$71682018000200003 \& \operatorname{lng}=$ es\&tlng=es.

Salado, L., Amavizca, S., Richart, R., y Rodríguez, R. (2020). Alfabetización digital de estudiantes universitarios en las modalidades presencial y virtual. Revista Electrónica de Investigación e Innovación Educativa-REIIE, 5(1), 30-47. http://doi.org/10.5281/zenodo.3629574.

Sánchez, H., Reyes, C. y Mejía, K. (2018). Manual de términos en investigación científica, tecnológica y humanística. https://www.urp.edu.pe/pdf/id/13350/n/libro-manual-de-terminos-eninvestigacion.pdf.

Siemens, G. (2004). A Learning Theory for the Digital Age. http://www.elearnspace.org/Articles/connectivism.htm.

Shopova, T. (2014). Digital Literacy of Students and its Improvement at the University. Journal on Efficiency and Responsibility in Education and Science, 7(2), 26-32. https://doi.org/10.7160/eriesj.2014.070201.

Valdivia, C. (8 de septiembre del 2020). Seminario virtual. La alfabetización de jóvenes y adultos en entornos digitales en el contexto de covid-19. https://lets.cinvestav.mx/Blog/TabId/134/ArtMID/543/ArticleID/111/SEMINARIO-VIRTUAL-Laalfabetizaci243n-de-j243venes-y-adultos-en-entornos-digitales-en-el-contexto-de-COVID-19.aspx.

Vásquez-Cano, E., López, E. y García-Garzón, E. (2017). Differences in basic digital competences between male and female university students of social science in Spain. International Journal of Educational Technology in Higher Education, 14(27), 1-16. https://link.springer.com/article/10.1186/s41239-017-0065-y.

Wang, X. y Li, B. (2019). Technostress among university teachers in higher education: A study using multidimensional person-environment misfit theory. Frontiers in Psychology, 10, 1-13. https://doi.org/10.3389/fpsyg.2019.01791 